\title{
ALGEBRAIC STRUCTURES OF QUANTUM PROJECTIVE FIELD THEORY RELATED TO FUSION AND BRAIDING. HIDDEN ADDITIVE WEIGHT.
}

\author{
Denis Juriev ${ }^{1}$ \\ Laboratoire de Physique Théorique de l'École Normale Supérieure, 24 rue Lhomond, \\ 75231 Paris Cedex 05, France ${ }^{2}$ \\ E mail: juriev@physique.ens.fr
}

The interaction of various algebraic structures, describing fusion, braiding and group symmetries in quantum projective field theory, is an object of an investigation in the paper. Structures of projective Zamolodchikov algebras, their representations, spherical correlation functions, correlation characters and envelopping QPFT-operator algebras, projective $\ddot{\mathrm{W}}$-algebras, shift algebras, infinite dimensional R-matrices $R_{\text {proj }}(u)$ and $R_{\text {proj }}^{*}(u)$ of the QPFT, braiding admissible QPFToperator algebras and projective $\mathrm{G}$-hypermultiplets are explored.

It is proved (in the formalism of shift algebras) that $\mathrm{sl}(2, \mathbb{C})$-primary fields are characterized by their projective weights and by the hidden additive weight, a hidden quantum number discovered in the paper.

The special attention is paid to various constructions of projective $G$-hypermultiplets (QPFT-operator algebras with $G$-symmetries).

PASC Numbers:

\footnotetext{
${ }^{1}$ On leave from Mathematical Division, Research Institute for System Studies, Russian Academy of Sciences, Moscow, Russia (e mail: juriev@systud.msk.su).

${ }^{2}$ Unité Propre du Centre National de la Recherche Scientifique associée à l'École Normale
} 


\section{INTRODUCTION.}

In view of the fact that an appearance of the representation theory depends on its applications, one might hope that a psychological change, which was provoked by a development of modern quantum field theory, would open new perspectives in the theory. Such hopes were confirmed by a lot of recent papers ${ }^{1-6}$. For example, accordingly to a program paper by G.Moore and N.Seiberg ${ }^{6}$ it is rather interesting to consider the concept of group being supplemented by various new concepts related to fusion and braiding. They considered some of those structures: vertex operator algebras, representations of bordism categories and quantum groups as well as relations between them.

An appearing of this paper is motivated by the following arguments. As one knows from the history of representation theory its primitive is a finite-dimensional Lie algebra $\operatorname{sl}(2, \mathbb{C})$ more than infinite-dimensional Kac-Moody and Virasoro algebras. So it is quite natural that the braiding-fusion structures connected with $\mathrm{sl}(2, \mathbb{C})$ should attract a special interest. In other words one should examine various algebraic structures of the quantum projective $(\mathrm{sl}(2, \mathbb{C})$-invariant) field theory but not only ones of the conformal or Kac-Moody-invariant field theories. Of course, such examinations should be based on the general theoretical results, which were obtained in papers on these theories.

These considerations explain the fact that structures of the quantum projective field theory (QPFT), connected with fusion and braiding, are investigated in this paper.

It is necassary to say some words about the tasks of the paper. Our task is not to introduce principally new structures, which have not been known. Moreover, the basic concepts of the paper are related to an operator product expansion (OPE), so the alternative but deeply connected approaches, related to quantum groups ${ }^{2-4}$ and bordism categories ${ }^{5}$, are not considered in the paper. Our task is to examine the OPE-structures of the QPFT in details,to describe some of them, which have no analogs in the conformal case, to write some explicit formulas, whose analogs in the conformal case have not be written in view of serious difficulties connected with the infinite-dimensionality of the Virasoro and Kac-Moody algebras.

The structures of projective Zamolodchikov algebras, their representations, spherical correlation functions and envelopping QPFT-operator algebras, projective $\ddot{\mathrm{W}}_{-}$ algebras, infinite dimensional R-matrices of the QPFT, braiding admissible QPFToperator algebras and projective $G$-hypermultiplets are explored.

It is proved (in the formalism of shift algebras) that $\mathrm{sl}(2, \mathbb{C})$-primary fields are characterized by their projective weights and by the hidden additive weight, a hidden quantum number discovered in the paper.

The paper contains 11 theorems, which have an original character. 


\section{FUSION IN THE QUANTUM PROJECTIVE FIELD THEORY: QPFT-OPERATOR ALGEBRAS.}

Definition 1: ${ }^{6-8}$ The QFT-operator algebra (operator algebra of quantum field theory) is the pair $\left(H, t_{i j}^{k}(\vec{x})\right)$, where $H$ is a linear space, $t_{i j}^{k}(\vec{x})$ is the $H$-valued tensor field on $\mathbb{C}^{n}$ or $\mathbb{R}^{n}$ of the fixed analytic class such that

$$
t_{i j}^{m}(\vec{x}-\vec{y}) t_{m k}^{l}(\vec{y})=t_{i m}^{l}(\vec{x}) t_{j k}^{m}(\vec{y})
$$

Remark: Let's introduce the operators $l_{\vec{x}}\left(e_{i}\right)$ such that

$$
l_{\vec{x}}\left(e_{i}\right) e_{j}=t_{i j}^{k}(\vec{x}) e_{k}
$$

then the following relations would hold

$$
l_{\vec{x}}\left(e_{i}\right) l_{\vec{y}}\left(e_{j}\right)=t_{i j}^{k}(\vec{x}-\vec{y}) l_{\vec{y}}\left(e_{k}\right) \quad \text { (operator product expansion (OPE)) }
$$

and

$$
l_{\vec{x}}\left(e_{i}\right) l_{\vec{y}}\left(e_{j}\right)=l_{\vec{y}}\left(l_{\vec{x}-\vec{y}}\left(e_{i}\right) e_{j}\right) \quad \text { (duality). }
$$

The QFT-operator algebra turns into the ordinary associative algebra iff $d t_{i j}^{k}(\vec{x})=$ 0 .

Definition 2: ${ }^{8}$ The QFT-operator algebra $\left(H, t_{i j}^{k}(u), u \in \mathbb{C}\right)$ is called the QPFToperator algebra iff

a) $H$ is the direct sum of the direct integral of The Verma modules $V_{a}$ over the Lie algebra $\operatorname{sl}(2, \mathbb{C})$ with the highest vectors $v_{a}$ and the highest weights $h_{a}$

b) the operator field $l_{u}\left(v_{a}\right)$ is primary, i.e.

$$
\left[L_{k}, l_{u}\left(v_{a}\right)\right]=(-u)^{k}\left(u \frac{\partial}{\partial u}+(k+1) h_{a}\right) l_{u}\left(v_{a}\right),
$$

where $L_{k}(k=-1,0,1)$ are the generators of the Lie algebra $\operatorname{sl}(2, \mathbb{C})$ : $\left[L_{k}, L_{j}\right]=(k-j) L_{k+j}$

c) the rule of secondaries' generation holds

$$
L_{-1} l_{u}(F)=l_{u}\left(L_{-1} F\right) .
$$

It maybe shown that QPFT-operator algebras are just the same as the QPFTsubalgebras in $\operatorname{Mat}_{N}(\operatorname{Vert}(\operatorname{sl}(2, \mathbb{C})))$, where $\operatorname{Vert}(\operatorname{sl}(2, \mathbb{C}))$ is the standard QPFToperator algebra - the algebra of the vertex operators for $\mathrm{sl}(2, \mathbb{C})$. It seems to be convenient as well as necessary to present the construction of the QPFT-operator algebra $\operatorname{Vert}(\operatorname{sl}(2, \mathbb{C})))$ below. To do it we need in a "model formalism".

Definition 3: ${ }^{8}$ The model of the Verma modules over the Lie algebra $\operatorname{sl}(2, \mathbb{C})$ is the representation of this algebra in the direct integral of the Verma modules $V_{h}$ 
The model admits several realizations. One of them is the BGG-realization ${ }^{8}$. The model space is the space of all holomorphic functions of two complex variables $t$ and $z$, where $z$ belongs to the complex plane $\mathbb{C}$ and $t$ belongs to the universal covering $\tilde{\mathbb{C}}^{*}$ of the complex plane without zero $\mathbb{C}^{*}=\mathbb{C} \backslash 0$. The generators of the Lie algebra $\operatorname{sl}(2, \mathbb{C})$ have the form

$$
L_{-1}=z, L_{0}=z \frac{\partial}{\partial z}-t \frac{\partial}{\partial t}, L_{1}=z\left(\frac{\partial}{\partial z}\right)^{2}-2 t \frac{\partial^{2}}{\partial t \partial z}
$$

The highest vector $v_{h}$ of the weight $h$ has the form $t^{-h}$. The second realization is one in the Fock space over the Laguerre deformation of the complex disk ${ }^{8}$. The model space is the same. the generators of the Lie algebra $\operatorname{sl}(2, \mathbb{C})$ have the form

$$
L_{-1}=z, L_{0}=z \frac{\partial}{\partial z}-t \frac{\partial}{\partial t}, L_{1}=z\left(\frac{\partial}{\partial z}\right)^{2}-2 t \frac{\partial^{2}}{\partial t \partial z}+t^{2} \frac{\partial}{\partial t}
$$

The highest vector $v_{h}$ of the weight $h$ has the form $t^{-h} F(-h, 2-2 h ; t z)$, where $F(a, b ; u)$ is the degenerate hypergeometric function. Other realizations of the model should be found in the Ref.8. It should be mentined that the realizations (5) and (6) are ones in the Fock spaces over the manifolds, on which $\mathrm{sl}(2, \mathbb{C})$ acts $^{8}$. Therefore, the structures of co-commutative coalgebras in the model are defined. The first structure is rather simple:

$$
\Delta\left(t^{\lambda} z^{k}\right)=\sum_{i+j=k} \int\left(t^{\rho} z^{i}\right) \otimes\left(t^{\lambda-\rho} z^{j}\right) d \rho
$$

but the second is interesting enough. In the realization (6) it has the same form as the first structure in the realization (5) but now the vectors $t^{\lambda} z^{k}$ do not generate the Verma module $V_{\lambda}$, so the formulas for the comultiplication contain all ClebschGordan coefficients for $\operatorname{sl}(2, \mathbb{C})$.

Now we shall be intereted in the hidden symmetries in the model. The ideology of Res-constructions of the models was proposed by I.M.Gelfand and A.V.Zelevinsky ${ }^{9}$. Res-construction in its general form means that the model of the representations of the Lie algebra $\mathfrak{g}$ is obtained as the restriction of an irreducible representation of an associative algebra, which contains the universal envelopping algebra $\mathcal{U}(\mathfrak{g})$ of the Lie algebra $\mathfrak{g}$. If one has the model of the representations of a Lie algebra $\mathfrak{g}$ and wants to obtain it by Res-construction he has to look for the hidden symmetries in the model, which form the necessary associative algebra together with the generators of the Lie algebra $\mathfrak{g}^{10}$. Let's denote $\mathcal{F}=\operatorname{span}\left(t^{\lambda} d t^{\mu}\right)$. Then the representation of the Lie algebra $\operatorname{sl}(2, \mathbb{C})$ in the model of the Verma modules over it can be extended to the action of the associative algebra $\mathcal{U}(\operatorname{sl}(2, \mathbb{C})) \ltimes \mathcal{F}^{8}$. The operator $T$, which represents the element $t$ of $\mathcal{F}$ in the realization (6) has the form

$$
T=t-\frac{\partial}{\partial z}
$$




$$
B_{\mu}=t^{-\mu} F\left(\mu, 2+\frac{\partial}{\partial t} ; t z\right),
$$

where $F(a, b ; u)$ is the normalized degenerate hypergeometric function

$$
F(a, b ; u)=\sum_{k \geq 0} u^{k} \frac{\Gamma(b-a) \Gamma(a+k)}{k ! \Gamma(a) \Gamma(b+k)} .
$$

The operator $B_{-1}$ is equal to $t^{2} \frac{\partial}{\partial t}+2 t-t^{2} z$. The operator $\nabla$ in the model of the Verma modules over the Lie algebra $\operatorname{sl}(2, \mathbb{C})$ is called a connection ${ }^{8}$ iff

$$
\left(\operatorname{ad}\left(L_{n}\right)-(n+1)(-T)^{n}\right) \nabla=0 ;[\nabla, f(T)]=f^{\prime}(T) .
$$

The operator $\nabla_{h}$, defined as $\nabla_{h}\left(f(T) L_{-1}^{k} v_{h}\right)=f^{\prime}(T) L_{-1}^{k} v_{h}$, is a connection. As it was shown in Ref.8 an arbitrary connection in the model of the Verma modules over the Lie algebra $\operatorname{sl}(2, \mathbb{C})$ coincides with one of the connections $\nabla_{h}$. Let $X$ be an operator in the model of the Verma modules over the Lie algebra $\mathrm{sl}(2, \mathbb{C})$, which commutes with all degrees of the operator $T$. The value of the operator $X$ in the point $u$ of the complex plane is the operator $X\left(u ; \nabla_{h}\right)=: \exp \left((T-u) \nabla_{h}\right): X$. The set of the operators $B_{\mu}\left(u ; \nabla_{h}\right)$ form the vertex operator fields in the model of the Verma modules over the Lie algebra $\operatorname{sl}(2, \mathbb{C})$. The explicit formulas for them are written in Ref.12. As it was shown in Ref.8 the set of the operator fields $z^{j} B_{\mu}\left(u ; \nabla_{h}\right)$ is closed under the operator product expansion. The corresponding QPFT-operator algebra is denoted by $\operatorname{Vert}(\operatorname{sl}(2, \mathbb{C}))$ and is called the algebra of vertex operators for the Lie algebra $\operatorname{sl}(2, \mathbb{C})$.

Let's consider the following linear operator in $\operatorname{Vert}(\operatorname{sl}(2, \mathbb{C}))$ :

$$
M_{a}: z^{j} B_{\mu}\left(u ; \nabla_{h}\right) \longmapsto z^{j} B_{\mu+a}\left(u ; \nabla_{h}\right) .
$$

Theorem 1: For all $A$ and $B$ from $\operatorname{Vert}(\operatorname{sl}(2, \mathbb{C}))$ the following relation holds

$$
A \cdot M_{a}(B)=M_{a}(A \cdot B) .
$$

Proof. Such relation should be verified on the basis of $\operatorname{Vert}(\operatorname{sl}(2, \mathbb{C}))$. Namely,

$$
\begin{aligned}
& z^{i} B_{\lambda}\left(u ; \nabla_{h}\right) M_{a}\left(z^{j} B_{\mu}\left(v ; \nabla_{g}\right)\right)=z^{i} B_{\lambda}\left(u ; \nabla_{h}\right) \cdot z^{j} B_{\mu+a}\left(v ; \nabla_{g}\right)= \\
& z^{i} B_{\lambda}\left(u ; \nabla_{h}\right) z^{j} B_{\mu}\left(v ; \nabla_{g}\right) B_{a}=M_{a}\left(z^{i} B_{\lambda}\left(u ; \nabla_{h}\right) \cdot z^{j} B_{\mu}\left(v ; \nabla_{g}\right)\right) .
\end{aligned}
$$

So the QPFT-operator algebra $\operatorname{Vert}(\operatorname{sl}(2, \mathbb{C}))$ admits a representation in the space $\operatorname{Fun}(\mathbb{R}, \mathbb{C}[z])$, whose elements $f(h, z)$ are the classes

$$
\int f(h, z) B_{\lambda}\left(u ; \nabla_{h}\right) d h /\left(\text { right multiplication on } B_{\lambda}\right) \text {. }
$$

As it was shown in Ref.8 the QPFT-operator algebras are just the same as the QPFT-operator subalgebras in $\operatorname{Mat}_{N}(\operatorname{Vert}(\operatorname{sl}(2, \mathbb{C})))$. Thus, the investigation of the fusion in the quantum projective field theory is reduced to an investigation of algebras of matrices with coefficients in the algebra of vertex operators for the Lie algebra $\operatorname{sl}(2, \mathbb{C})$. Another approach to the fusion in the quantum projective field theory is presented in the following paragraph. 


\section{AN ALTERNATIVE APPROACH TO THE FUSION IN THE QUAN- TUM PROJECTIVE FIELD THEORY: SHIFT ALGEBRAS.}

Let's consider the space $T=\mathbb{C}\left[x, x^{-1}, y\right]$ where the variables $x$ and $y$ do not commute: $x^{m} P(y)=P(y-m) x^{m}$. The space $T$ maybe considered as the algebra of weighted shift operators on $\mathbb{Z}$ (cf.Ref.13). One should mention that $[x, y]=x$ so $T$ is a representation space for the Lie algebra aff of affine vector fields on $\mathbb{R}$. Such representation maybe extended to a representation of the Lie algebra $W_{1}$ of formal vector fields on $\mathbb{R}$ by the adjoint action of the elements

$$
a_{-1}=x, a_{0}=y, a_{1}=x^{-1} y(y-1), \ldots a_{n}=x^{-n} y \ldots(y-n), \ldots
$$

It should be marked that $T=\mathbb{C}\left[x, x^{-1}\right] \otimes \operatorname{span}\left(a_{0}, a_{1}, a_{2}, \ldots a_{n}, \ldots\right)$ and

$$
a_{i} a_{j}=x a_{i+j+1}+(i+1) a_{i+j} .
$$

The elements $a_{-1}, a_{0}, a_{1}$ generate the Lie algebra sl $(2, \mathbb{C})$. Let's consider the authomorphisms $H_{h}$ of $T$

$$
H_{h}(a)=x^{-h} a x^{h}, \quad h \in \mathbb{R},
$$

which are internal for $z \in \mathbb{Z}$. Let also denote

$$
a_{n}(h)=H_{h}\left(a_{n}\right) .
$$

Then

$$
\begin{gathered}
a_{-1}(h)=x, a_{0}(h)=y+h, a_{1}(h)=x^{-1}(y+h)(y+h-1), \ldots \\
a_{n}(h)=x^{-n}(y+h) \ldots(y-n+h), \ldots
\end{gathered}
$$

so that $T=\mathbb{C}\left[x, x^{-1}\right] \otimes\left(a_{0}(h), a_{1}(h), a_{2}(h), \ldots\right)$ and

$$
a_{i}(h) a_{j}(h)=x a_{i+j+1}(h)+(i+1) a_{i+j}(h) .
$$

The elements $a_{i}(h)(i=-1,0,1)$ generate the Lie algebra $\mathrm{sl}(2, \mathbb{C})$. One should mention that

$$
H_{h} H_{h^{\prime}}=H_{h+h^{\prime}}
$$

so that $H_{h}=\exp (h d)$. Let's consider the operator $\xi: T \mapsto T$ such that 
Then $\xi$ is a derivation of $T$. Moreover, $\xi$ commutes with all $H_{h}$ and, therefore, with $d$. Let's extend the algebra $T$ by the variable $d$ to the algebra $T^{\prime}$ such that $[x, y]=x,[x, d]=0,[d, y]=1 ; \xi$ acts in $T^{\prime}$ by derivations. Let's also consider the space $\tilde{T}^{\prime}$ in which we admit formal series by variables $x, x^{-1}, y, d$.

Definition 4: An element $v_{\mu} \in \tilde{T}^{\prime}$ is called basic of the weight $\mu$ iff

$$
\left(\operatorname{ad}\left(a_{n}\right)-(-1)^{n}(\xi+n \mu) v_{\mu}=0 .\right.
$$

The elements $x^{n} v_{\mu}, n \geq 0$ generate the family of a basic element $v_{\mu}$.

We shall denote the subspace of $\tilde{T}^{\prime}$ of the elements from $\mathbb{C}\left[\left[x, x^{-1}, y\right]\right] H_{h}$ by $T_{h}^{\prime}$.

Theorem 2: There exists the unique up to a multiple basic element of the weight $\mu$ in the space $\tilde{T}_{h}^{\prime}$, which will be denoted by $v_{\mu, h}$. The basic element $v_{\mu, h}$ has the form

$$
v_{\mu, h}=s^{+}(x, y ; \mu, h) H_{h}=H_{h} s^{-}(x, y ; \mu, h),
$$

( $x$ stands on the left side from $y$ )

$$
\begin{gathered}
s^{+}(x, n ; \mu, h)=s_{n}^{+}(x ; \mu, h) \\
s^{-}(x, n ; \mu, h)=s_{n}^{-}(x ; \mu, h) \\
s_{n+1}^{+}(x)=\left(s_{n}^{+}(x)\right)^{\prime}+\left(1-a^{+} / x\right) s_{n}^{+}(x) \\
s_{n+1}^{-}(x)=\left(s_{n}^{-}(x)\right)^{\prime}+\left(1-a^{-} / x\right) s_{n}^{-}(x) \\
a^{+}=a^{-}=\mu-h \\
\left(s_{n}^{+}\right)^{\prime \prime \prime}+A_{n}^{+}(x)\left(s_{n}^{+}\right)^{\prime \prime}+B_{n}^{+}(x)\left(s_{n}^{+}\right)^{\prime}+C_{n}^{+}(x) s_{n}^{+}=0 \\
\left(s_{n}^{-}\right)^{\prime \prime \prime}+A_{n}^{-}(x)\left(s_{n}^{-}\right)^{\prime \prime}+B_{n}^{-}(x)\left(s_{n}^{-}\right)^{\prime}+C_{n}^{-}(x) s_{n}^{-}=0 \\
s_{n}^{+}(x) \sim_{x \rightarrow 0} x^{-n}, s_{n}^{-}(x) \sim_{x \rightarrow 0} x^{-n} \\
A_{n}^{+}(x)=2+\left(2+b_{n}^{-}-a^{-}\right) / x \\
B_{n}^{+}(x)=1+\left(2+b_{n}^{+}-a^{+}+c^{+}\right) / x+\left(d_{n}^{+}-a^{+} b_{n}^{+}\right) / x^{2} \\
C_{n}^{+}(x)=\left(c^{+}-e_{n}^{+}\right) / x+\left(a^{+}+d_{n}^{+}-a^{+} c^{+}\right) / x^{2}+a^{+}\left(b_{n}^{+}-d_{n}^{+}\right) / x^{3} \\
A_{n}^{-}\left(2+b_{n}^{-}-a^{-}\right) / x+\left(d_{n}^{-}-a^{-} b_{n}^{-}\right) / x^{2}
\end{gathered}
$$




$$
\begin{aligned}
& b_{n}^{+}=2 n, c^{+}=\mu+h, d_{n}^{+}=n(n+1), e_{n}^{+}=n(n+1)-2 h \\
& b_{n}^{-}=2(n+h), c^{-}=\mu+h, d_{n}^{-}=(n+2 h)(n+1), e_{n}^{-}=n(n+1)
\end{aligned}
$$

Proof. The formulas for $v_{\mu, h}$ are obtained by the direct but tedious computations, so they should be omitted.

The operator $\xi$ generates the 1 -parametric family $\alpha_{u}\left(u \in \mathbb{C}^{*}\right)$ of automorphisms of $\tilde{T}^{\prime}$. It should be mentioned that the product of two basic elements $v_{1}$ and $v_{2}$ is ill-defined, because they are the formal series. Nevertheless, one may regularize their product by the automorphism $\alpha_{u}$. The product

$$
\left(\alpha_{u} v_{1}\right) v_{2}=\sum_{w-\text { basic }} u^{k(w)} P_{w}(x) w
$$

is correctly defined Laurent series. Such OPE will be called the regularization of product $v_{1} v_{2}(\mathrm{RP})$.

Definition 5: A set of families of basic elements in $\operatorname{Mat}_{N}(\mathbb{C}) \otimes \tilde{T}^{\prime}$, which is closed under the RP for all $u$, is called a shift algebra.

Theorem 3: There exists a strict functor from the category of the QPFToperator algebras to the category of shift algebras and vice versa.

Proof. One should compare formulas (22-28) with ones for the vertex operator fields in the model of the Verma modules over the Lie algebra $\operatorname{sl}(2, \mathbb{C}){ }^{12}$ and then use the results of the second paragraph.

It will be very interesting to give an interpretation of shift algberas in terms of geometric quantization in a way analogous to Ref.14.

Remark: If $v_{\mu, h}$ is the basic of the weight $\mu$, then the operator field $u^{a} \alpha_{u}\left(v_{\mu, h}\right)$, where $a=a^{-}=a^{+}$, is $\operatorname{sl}(2, \mathbb{C})$-primary.

Remark: (on the hidden additive weight of the $\mathrm{sl}(2, \mathbb{C})$-primary fields). From the fact that $\tilde{T}_{h}^{\prime} \tilde{T}_{g}^{\prime}=\tilde{T}_{h+g}^{\prime}$ the additivity of the hidden quantum number $h$ of the $\operatorname{sl}(2, \mathbb{C})$-basics $v_{\mu, h}$ follows.

\section{PROJECTIVE $\ddot{W}$-ALGEBRAS.}

One of the most intriguing object of the quantum conformal field theory is Walgebra ${ }^{15}$. In this paragraph we inwestigate objects, which are similar to $\mathrm{W}_{-}$ algebras in several properties but differ in others (cf.Ref.16) so they will be called projective $\ddot{\mathrm{W}}$-algebras ("double $\ddot{\mathrm{u}}$-algebras").

Definition 6: Let $H$ be an arbitrary QPFT-operator algebra. The associative algebra generated in the space $H$ by operators $a_{t, a}$

$$
\int a_{t, a} u^{t} d t=V_{a}(u),
$$

where $\left\{V_{a}(u)\right\}$ is the set of all primary fields in the QPFT-operator algebra $H$, is called the associated projective $\ddot{\mathrm{W}}$-algebra and is denoted by $\ddot{\mathrm{W}}(H)$.

As follows from the results of Ref.8 each projective $\ddot{\mathrm{W}}$-algebra is a subalgebra in $\operatorname{Mat}_{N}\left(\ddot{\mathrm{W}}_{\text {proj }}\right)$, where $\ddot{\mathrm{W}}_{\text {proj }}=\ddot{\mathrm{W}}(\operatorname{Vert}(\mathrm{sl}(2, \mathbb{C})))$ is the universal projective $\ddot{\mathrm{W}}-$ 
Theorem 4: The universal projective $\ddot{W}$-algebra $\ddot{W}_{\text {proj }}$ is the algebra of the linear operators in the model of the Verma modules over the Lie algebra $\operatorname{sl}(2, \mathbb{C})$. Its generators $a_{t, \mu, h}$ have the form

$$
\begin{aligned}
& a_{t, \mu, h}=\bigoplus_{k} a_{t, \mu, h}^{k}, \quad a_{t, \mu, h}^{k}: V_{h-t-\mu+k} \longmapsto V_{h} \\
& a_{t, \mu, h}^{k}=z^{k} F_{k}\left(z \frac{\partial}{\partial z} ; \mu, h-t-\mu+k, h\right), \quad k \geq 0 \\
& a_{t, \mu, h}^{k}=\left(\frac{\partial}{\partial z}\right)^{k} F_{k}\left(z \frac{\partial}{\partial z} ; \mu, h-t-\mu+k, h\right), \quad k \leq 0,
\end{aligned}
$$

where the functions $F_{k}(x ; \mu, g, h)=\sum_{j} F_{k j}(\mu, g, h) \delta(x-j)$ obey the difference hypergeometric equations

$$
P_{ \pm}(x) \Delta^{2} F+Q_{ \pm}(x) \Delta F+R_{ \pm} F=0
$$

the signs correspond to the positive or negative $k$,

$$
\begin{aligned}
& P_{+}(x)=(x+k+2)(x+k+2 h+1) \\
& P_{-}(x)=(x+2)(x+2 h+k+1) \\
& Q_{+}(x)=2(k+1+h-g) x+(k+2)(k+1+2 h)-2 g+ \\
&(k+h-g+\mu)(k-\mu+h-g+1) \\
& Q_{-}(x)=2(1+h-g) x+2 h k+2(1+2 h-g)+ \\
& \quad(k+h-g+\mu)(k-\mu+h-g+1) \\
& R_{+}=(k+h-g+\mu)(k+1-\mu+h-g) \\
& R_{-}=(k+h-g+\mu)(k+1-\mu+h-g)
\end{aligned}
$$

the functions $F_{k}$ are connected by the following equations

$$
\begin{aligned}
& F_{k}(x)=(k-\mu+h-g)^{-1} \Delta F_{k-1}(x), \quad k \geq 0 \\
& F_{k}(x)=(k-\mu+h-g)^{-1}\left((x+1) F_{k-1}(x+1)-(x+k) F_{k-1}(x)\right), \quad k \leq 0 .
\end{aligned}
$$

Proof. The theorem follows from the explicit formulas for the vertex operator fields in the model of the Verma modules over $\operatorname{sl}(2, \mathbb{C})^{12}$.

\section{BRAIDING IN THE QUANTUM PROJECTIVE FIELD THEORY: PROJECTIVE ZAMOLODCHIKOV ALGEBRAS.}

The most interesting fact is that the braiding in the quantum projective field theory is naturally described in the same terms as fusion, i.e. in terms of vertex operator fields in contrast to the quantum conformal field theory, where there are 
As it was shown in Ref.8 the Sklyanin-Faddeev-Zamolodchikov relations ${ }^{2,18}$ for the vertex operator fields $B_{\lambda}\left(u ; \nabla_{h}\right)$ hold:

$$
B_{\lambda_{1}}\left(u ; \nabla_{h_{1}}\right) B_{\lambda_{2}}\left(v ; \nabla_{h_{2}}\right)=\sum\left(R_{\mathrm{proj}}(u-v)_{\lambda_{1} \lambda_{2} h_{1} h_{2}}^{\lambda_{3} \lambda_{4} h_{3} h_{4}} B_{\lambda_{3}}\left(v ; \nabla_{h_{3}}\right) B_{\lambda_{4}}\left(u ; \nabla_{h_{4}}\right) .\right.
$$

The R-matrix $R_{\text {proj }}(u)$ obeys the quantum Yang-Baxter equation ${ }^{2,19}$ :

$$
R_{\text {proj }}(v-u)_{\alpha \beta}^{\delta \varepsilon} R_{\text {proj }}(v)_{\varepsilon \gamma}^{\zeta \eta} R_{\text {proj }}(u)_{\delta \zeta}^{\theta \varkappa}=R_{\text {proj }}(u)_{\beta \gamma}^{\delta \varepsilon} R_{\text {proj }}(v)_{\alpha \delta}^{\theta \zeta} R_{\text {proj }}(v-u)_{\zeta \varepsilon}^{\varkappa \eta}
$$

where $\alpha, \beta, \gamma, \delta, \varepsilon, \zeta, \eta, \theta, \varkappa \in \mathbb{R}^{2}$.

Remark:

$$
\left(R_{\text {proj }}(u)\right)_{\lambda_{1} \lambda_{2} h_{1} h_{2}}^{\lambda_{3} \lambda_{4} h_{3} h_{4}} \neq 0 \text { only if } h_{1}=h_{3}
$$

\section{Theorem 5:}

$$
\left(R_{\text {proj }}(u)\right)_{\lambda_{1} \lambda_{2}+\mu h_{1} h_{2}}^{\lambda_{3} \lambda_{4}+\mu h_{3} h_{4}}=\left(R_{\text {proj }}(u)\right)_{\lambda_{1} \lambda_{2} h_{1} h_{2}}^{\lambda_{3} \lambda_{4} h_{3} h_{4}}
$$

Proof of the theorem is analogous to one of the theorem 1.

Definition 7: ${ }^{18,19}$ Zamolodchikov algebra, correspondinig to the R-matrix $R(u)_{k l}^{i j}$, obeying the quantum Yang-Baxter equation, is the set of formal generators $A_{i}(u)$ such that

$$
A_{i}(u) A_{j}(v)=R(u-v)_{i j}^{k l} A_{k}(v) A_{l}(u)
$$

Two Zamolodchikov algebras $\mathcal{A}=\left\{A_{i}(u)\right\}$ and $\mathcal{A}^{\prime}=\left\{A_{i}^{\prime}(u)\right\}$ will be called equivalent iff the corresponding $\mathrm{R}$-matrices are equivalent, i.e.

$$
R^{\prime}(u)_{i j}^{k l}=R(u)_{p r}^{m n} C_{m}^{k} C_{n}^{l} C_{i}^{p} C_{j}^{q}
$$

for some invertible matrix $C_{b}^{a}$.

By the representation of the Zamolodchikov algebra we mean the representation of formal generators $A_{i}(u)$ by the analytic operator fields so that the product $A_{i}(u) A_{j}(v)$ exists if $|u| \gg|v|$ and maybe analytically extended to $\mathbb{C}^{2}$. After the analytic continuation the relation (39) should hold.

Theorem 6: The set of the primary vertex operator fields in the QPFT-operator algebra $\operatorname{Vert}(\operatorname{sl}(2, \mathbb{C})))$ is the Zamolodchikov algebra.

Remark: It is an interesting problem to formulate the Cherednik-type representation ${ }^{20}$ of the $\mathrm{R}-$ matrix $R_{\text {proj }}(u)$ and the Zamolodchikov algebra of the theorem 6.

Definition 8: The Zamolodchikov algebra $\mathcal{A}=\left\{A_{i}(u)\right\}$ is called the projective Zamolodchikov algebra iff there exists the linear space $H$, which can be expanded in the direct sum or the direct integral of the Verma modules over the Lie algebra 


$$
\left[L_{k}, A_{i}(u)\right]=(-u)^{k}\left(u \frac{\partial}{\partial u}+(k+1) s_{i}\right) A_{i}(u) .
$$

It is an interesting problem to describe the class of projective Zamolodchikov algebras in the internal terms.

Hypothesis: Each Zamolodchikov algebra maybe imbed into a projective Zamolodchikov algebra.

By the representation of the projective Zamolodchikov algebra we mean a representation of its generators by the primary fields. One of the characteristics of a representation of the projective Zamolodchikov algebra is its weight $\left(s_{1}, \ldots s_{n}\right)$, where $s_{i}$ are the weights of the primary operator fields, corresponding to the generators $A_{i}(u)$.

Lemma: Let $H$ be a linear space, which can be expanded into a direct sum or a direct integral of the Verma modules over the Lie algebra $\operatorname{sl}(2, \mathbb{C}) . \mathcal{V}=\left\{V_{i}(u)\right\}$ is the set of primary operator fields in $H$. The QFT-operator algebra generated by $V_{i}(u)$ is a QPFT-operator algebra.

Proof. One should mention that the space $\bigoplus<z^{j} V_{a}(u)>$, where $\left\{V_{A}(u)\right\}$ is the set of all primary fields in $H$, is closed onder the OPE and so it is a QPFToperator algebra. Also if the OPE of two primary fields contains a secondary of the third one then it contains this primary field ${ }^{21}$. So the QFT-operator algebra generated by $\left\{V_{i}(u)\right\}$ is a QPFT-operator subalgebra in $\bigoplus<z^{j} V_{a}(u)$ : $V_{a}(u)$ - all primary operator fields $>$.

Theorem 7: Let $\mathcal{A}$ be a projective Zamolodchikov algebra represented in $H$. The QFT-operator algebra generated by $A_{i}(u)$ is a QPFT-operator algebra.

Proof. The statement of the theorem is a sequence of the previous lemma.

Definition 9: Let $\mathcal{A}$ be a projective Zamolodchikov algebra represented in $H$. The QPFT-operator algebra constructed in the previous theorem will be called the envelopping QPFT-operator algebra of $(\mathcal{A}, H)$ and will be denoted by $\operatorname{QPFT}(\mathcal{A}, H)$.

It is an interesting question whether exists the universal envelopping QPFToperator algebra of a projective Zamolodchikov algebra $\mathcal{A}$.

Different representations of the fixed projective Zamolodchikov algebra may have the same envelopping QPFT-operator algebras, therefore, a natural problem of the representation theory of projective Zamolodchikov algebras is to describe the possible envelopping QPFT-operator algebras.

Definition 10: A QPFT-operator algebra is called braiding admissible QPFToperator algebra (b.a.QPFT-operator algebra) iff there exists a basis of primary operator fields in it, which forms a projective Zamolodchikov algebra.

It is an interesting question to find the conditions, which extract the b.a.QPFToperator algebras from all QPFT-operator algebras.

One has a functor from the category of representations of the projective Zamolodchikov algebras to the category of b.a.QPFT-operator algebras. What are the projective Zamolodchikov algebras, which have a common b.a.QPFT-operator algebra?

Now we shall describe an important object connected with representations of projective Zamolodchikov algebras. It is analogous to spherical functions in the group representation theory ${ }^{22}$ and so will be called a spherical correlation function.

Definition 11: Let $\mathcal{A}$ be a projective Zamolodchikov algebra represented in the 
vector $v_{a}$ and a highest weight $h_{a}$. The spherical correlation functions of generators $A_{i_{1}}\left(u_{1}\right), \ldots A_{i_{n}}\left(u_{n}\right)$ are

$$
<A_{i_{1}}\left(u_{1}\right) \ldots A_{i_{n}}\left(u_{n}\right)>_{a b}=<v_{a}\left|A_{i_{1}}\left(u_{1}\right) \ldots A_{i_{n}}\left(u_{n}\right)\right| v_{b}>
$$

Theorem 8: A representation of a projective Zamolodchikov algebra can be constructed from the spherical correlation functions

$$
<A_{i}(u)>_{a b}
$$

Proof. It should be mentioned that a representation of a projective Zamolodchikov algebra can be characterized by its weight $\left(s_{1}, \ldots s_{n}\right)$ and the set of the constants

$$
c_{k, a b}=\operatorname{Proj}_{V_{b}}\left(\left(\left.A_{k}(u)\right|_{V_{a}}\right) / V_{s_{k}}\left(u ; h_{a}, h_{b}\right)\right.
$$

where $V_{s}(u ; h, g)$ is the standard primary operator field of the weight $s$ acting from the Verma module $V_{h}$ to the Verma module $V_{g}{ }^{8,12}$. Let us mark that

$$
<v_{a}\left|V_{s_{k}}\left(u ; h_{a}, h_{b}\right)\right| v_{b}>=u^{-s_{k}+\left(h_{b}-h_{a}\right)} .
$$

So one can reconstruct the weight $s_{k}$ and the constant $c_{k, a b}$ from the correlation function $<A_{k}(u)>_{a b}$.

Let's consider the matrix generators $E_{a b}$. Using them one can collect spherical correlation functions in the following expressions

$$
<A_{i_{1}}\left(u_{1}\right) \ldots A_{i_{m}}\left(u_{m}\right)>:=\sum_{a, b}<A_{i_{1}}\left(u_{1}\right) \ldots A_{i_{m}}\left(u_{m}\right)>_{a b} E_{a b} .
$$

Definition 12: Let $\mathcal{A}$ be a projective Zamolodchikov algebra represented in $H$ by primary operator fields $A_{i}(u)$. The correlation character of generators $A_{i_{1}}\left(u_{1}\right), \ldots A_{i_{m}}\left(u_{m}\right)$ is

$$
\operatorname{Tr}<A_{i_{1}}\left(u_{1}\right) \ldots A_{i_{m}}\left(u_{m}\right)>
$$

It should be mentioned that as a rule the trace does not exist in the naïve sense so one should consider it either as a trace in a suitable von Neumann algebra or as a generalized function (using some smoothing by $u_{k}$ ), it is possible also to regularize the trace in some way, f.e. by an insertion of the multiple $q^{L_{0}}$.

It is an interesting question whether a representation of the projective Zamolodchikov algebra can be reconstructed from the correlation character.

VI. AN ALTERNATIVE APPROACH TO THE BRAIDING IN THE QUANTUM PROJECTIVE FIELD THEORY: INFINITE DIMENSIONAL PARAMETRIC R-MATRIX $R_{\text {proj }}^{*}(u)_{h, h^{\prime}}$. 
Theorem 9: For all $h$ and $h^{\prime}$ the forllowing relation holds (cf.Ref.6,23):

$$
v_{\lambda, h}(u) v_{\lambda^{\prime}, h^{\prime}}(v)=\left(R_{\text {proj }}^{*}(u / v)\right)_{h, h^{\prime} ; \lambda \lambda^{\prime}}^{\mu \mu^{\prime}} v_{\mu, h}(v) v_{\mu^{\prime}, h^{\prime}}(u),
$$

the matrix $R_{\text {proj }}^{*}(u)_{h, h^{\prime}}$ obeys the following Yang-Baxter-type equation

$$
\begin{aligned}
& R_{\text {proj }}^{*}(u / v)_{h, g ; ;_{\alpha \beta}}^{\delta \varepsilon} R_{\text {proj }}^{*}(u / w)_{g, f ;} \underset{\varepsilon \gamma}{\zeta \eta} R_{\text {proj }}^{*}(v / w)_{h, g ; \delta \zeta} \underset{\delta \varkappa}{\theta \varkappa}= \\
& R_{\text {proj }}^{*}(v / w)_{g, f ;{ }_{\beta \gamma}}{ }_{\beta \varepsilon} R_{\text {proj }}^{*}(u / w)_{h, g ; \alpha \delta}{ }_{; \zeta} R_{\text {proj }}^{*}(u / v)_{h, f ; \zeta \varepsilon}^{\varkappa \eta} .
\end{aligned}
$$

Proof. the theorem follows from the fact that for all $h, h^{\prime}, u, v$ the operators $v_{\lambda, h}(v) v_{\lambda^{\prime}, h^{\prime}}(u)$ form a basis in the space $\tilde{T}_{h+h^{\prime}}^{\prime}$. The dependence of the $\mathrm{R}$-matrix on the quotient $u / v$ follows from properties of the transformations of fields under the action of $\xi$.

\section{PROJECTIVE G-HYPERMULTIPLETS.}

Definition 13: Let $G$ be a group. A QPFT-operator algebra will be called a projective $G$-hypermultiplet iff $G$ acts in the space $H$ of the algebra by automorphisms and $G$ commutes with $\operatorname{sl}(2, \mathbb{C})$.

It can be easily shown that the spaces of the highest vectors of a fixed highest weight in a projective $G$-hypermultiplet form representations of $G$. Such representations will be called multiplets of projective $G$-hypermultiplet.

Let's construct several examples of projective hypermultiplets.

Definition 14: Let $\mathfrak{g}$ be a Lie algebra, $G$ - a group of its automorphisms. Let $t$ be a representation of $\mathfrak{h}, g-$ an element of $G$. We'll denote by $t^{g}$ the representation of $\mathfrak{h}$, which is defined as follows

$$
A \longmapsto t(g(A)) .
$$

A representation $t$ of $\mathfrak{h}$ will be called $G$-stable iff $t^{g}$ are equivalent to each other for all $g$.

Lemma: Let $\mathfrak{h}$ be a Lie algebra with $\widetilde{\operatorname{PSl}}(2, \mathbb{R})$ as an automorphism group. Then the space of an arbitrary $\widetilde{\mathrm{PSl}}(2, \mathbb{R})$-stable representation $t$ of $\mathfrak{h}$ admits a structure of $\widetilde{\mathrm{PSl}}(2, \mathbb{R})$-representation $T$ so that $t(g(A))=T(g) t(A) T\left(g^{-1}\right)$.

Definition 15: (Cf.Ref.24) Let $\mathfrak{h}$ be an arbitrary Lie algebra with basis $X^{m}$ and commutation relations

$$
\left[X^{m}, X^{n}\right]=c_{p}^{m n} X^{p}
$$

then the truncated current algebra ${ }^{t} \hat{\mathfrak{h}}$ over $\mathfrak{h}$ is the Lie algebra with basis $T_{r}^{m}, r \geq 0$, and the commutation relations 
One should mention that an action of $\operatorname{sl}(2, \mathbb{C})+\mathfrak{h}^{\mathbb{C}}$ in ${ }^{t} \hat{\mathfrak{h}}^{\mathbb{C}}$ defined as

$$
L_{k}\left(T_{r}^{m}\right)=r T_{r+k}^{m}, \quad X^{m}\left(T_{r}^{n}\right)=c_{l}^{m n} T_{r}^{l}
$$

maybe extended to a representation of $\widetilde{\mathrm{PSl}}(2, \mathbb{R}) \times H$ in ${ }^{t} \hat{\mathfrak{h}} \mathbb{C}$ by automorphisms.

Theorem 10: Let $t$ be an arbitrary $\widetilde{\mathrm{PSl}}(2, \mathbb{R}) \times H$-stable representation of ${ }^{t} \hat{\mathfrak{h}}^{\mathbb{C}}$ and suppose that the corresponding representation $T$ of $\operatorname{sl}(2, \mathbb{C})$ belongs to the category $\mathcal{O}^{25}$. Then the operator fields (currents)

$$
T^{m}(u)=\sum_{r \geq 0} T_{r}^{m} u^{-1+r}+\text { regular terms }
$$

generate a projective $H$-hypermultiplet.

Proof. The theorem folows from the lemma before the theorem 7 and the fact that an induced action of $H$ on the QPFT-operator algebra, generated by the currents $T^{m}(u)$, preserves the structure of the QFT-operator algebra and $\mathrm{sl}(2, \mathbb{C})$ action.

Let's now consider another example of projective $G$-hypermultiplet.

Theorem 11: Let $\mathcal{A}$ be a projective Zamolodchikov algebra with a group $G$ of its automorphisms and $H-$ an arbitrary space, where $\mathcal{A}$ is represented. Let $K$ be a subgroup of $G$ such that $K \subseteq \operatorname{Aut}(\operatorname{QPFT}(\mathcal{A}, H))$. Then the QPFT-operator algebra $\operatorname{QPFT}(\mathcal{A}, \hat{H})$, where $\hat{H}=\operatorname{Ind}_{K}^{G}(H)$, is a b.a.projective $G$-hypermultiplet.

\section{CONCLUSIONS.}

Thus, various structures of the quantum projective field theory related to fusion and braiding were explored. projective Zamolodchikov algebras, their representations, spherical correlation functions and correlation characters, envelopping QPFT-operator algebras, projective $\ddot{\mathrm{W}}$-algebras, shift algebras, infinite dimensional R-matrices, projective $G$-hypermultiplets are among them. various approaches to fusiona and braiding are presented.

It is proved (in the formalism of shift algebras) that $\mathrm{sl}(2, \mathbb{C})$-primary fields are characterized by their projective weights and a hidden quantum number discovered in the paper - the hidden additive weight.

\section{ACKNOWLEDGEMENTS.}

The author thanks A.Yu.Morozov, A.A.Rosly, A.S.Losev, M.A.Olshanetsky, A.Marshakov, A.Gerasimov and other participants of the Seminar on Quantum field Theory in ITEP (Moscow) for an attention and remarks.

\section{REFERENCES}

1 E.Witten, "Physics and geometry", Rep. 100th Anniversary A.M.S., 1988; L.D.Faddeev, Preprint SPhT 82/76, CEN Saclay, 1982; A.A.Belavin, A.M.Polyakov, and A.B.Zamolodchikov, Nucl. Phys. B241, 333 (1984); A.B.Zamolodchikov, and Al.B.Zamolodchikov, 
2 E.K.Sklyanin, and L.D.Faddeev, Dokl. Akad. Nauk SSSR 243(6) 1430 (1978).

3 N.Yu.Reshetikhin, LOMI Preprint E-4-87, 1988; N.Yu.Reshetikhin, L.A.Takhtajan, and L.D.Faddeev, Algebra Anal. 1(1), 178 (1989); L.C.Biedenharn, "An overview of quantum groups", Proc. 18th Intern. Colloq. Group Methods, Moscow, 1990; N.Yu.Reshetikhin, and M.A.Semenov-Tian-Shansky, Lett. Math. Phys. 19, 133 (1990).

4 V.G.Drinfeld, "Quantum groups", Proc. Intern. Congr. Math., Berkeley, 1986; Yu.I.Manin, Preprint CRM-1561, Montreal, 1988.

5 G.Segal, Preprint MPI/87-85, 1988; E.Witten, Commun. Math. Phys. 113, 529 (1988); M.Kontsevich, unpublished notes, 1988; L.Alvarez-Gaume, C.Gomez, G.Moore, and C.Vafa, Nucl. Phys. B303, 455 (1988).

6 G.Moore, and N.Seiberg, Commun. Math. Phys. 123, 177 (1989).

7 A.Z.Patashinskii, and V.L.Pokrovskii, "Fluctuation theory of phase transitions", Pergamon Press, 1978; R.E.Borcherds, Proc. Nat'l Acad. Sci. USA 83, 3068 (1986); I.Frenkel, J.Lepowsky, and A.Meurman, "Vertex operator algebras and the Monster", New York, 1988; P.Goddard, "Meromorphic conformal field theory" in "Infinite dimensional Lie algebras and groups", World Scientific, 1989.

8 D.V.Juriev, Algebra Anal. 3(3), 197 (1991), Uspekhi Matem. Nauk 46(4), 115 (1991); S.A.Bychkov, and D.V.Juriev, Teor. Matem. Fiz. 97(3) (1993).

9 I.M.Gelfand, and A.V.Zelevinsky, Funkt. anal. i ego prilozh. 18(3), 14 (1984).

10 D.E.Flath, and L.C.Biedenharn, Canad. Math. J. 37(4), 710 (1985).

11 D.Juriev, Lett. Math. Phys. 21, 133 (1991).

12 D.Juriev, Lett. Math. Phys. 22, 141 (1991).

13 Yu.D.Latushkin, and A.M.Stepin, Uspekhi Matem. Nauk 46(2), 85 (1991).

14 A.Yu.Alekseev, and S.L.Shatashvili, Commun. Math. Phys. 128, 197 (1990).

15 F.A.Bais, P.Bouwknegt, M.Surridge, and K.Schoutens, Nucl. Phys. B304, 348 (1988); I.Bakas, Commun. Math. Phys. 123, 627 (1989); O.D.Ovsienko, and V.Yu.Ovsienko, Adv. Soviet Math. 2, 221 (1991).

16 A.Bilal, Preprint PUPT-1434; hep-th/9312108; Preprint PUPT-1446; hep-th/9401167.

17 A.Yu.Morozov, Preprint ITEP 89/148, 1989; A.O.Radul, Funkt. anal. i ego prilozh. 25(1), 33 (1991).

18 E.K.Sklyanin, L.A.Takhtajan, and L.D.Faddeev, Teor. Matem. Fiz. 40, 194 (1979); A.B.Zamolodchikov, Pis'ma ZhETP 25(10), 13 (1977).

19 L.A.Takhtajan, and L.D.Faddeev, Uspekhi Matem. Nauk 34(5), 13 (1979).

20 I.V.Cherednik, Dokl. Akad. Nauk SSSR 249(5), 1095 (1979).

21 A.A.Belavin, A.M.Polyakov, and A.B.Zamolodchikov, Nucl. Phys. B241, 333 (1984).

22 D.P.Zhelobenko, and A.I.Schtern, "Representations of Lie groups", Moscow, Nauka, 1983.

23 G.Moore, and N.Yu.Reshetikhin, Preprint IASSNS-HEP-89/18, 1989.

24 V.G.Kac, "Infinite dimensional Lie algebras", Cambridge, 1985.

25 I.N.Bernstein, I.M.Gelfand, and S.I.Gelfand, Funkt. anal. i ego prilozh. 5(1), 1 (1971). 